\title{
Fast-and-Smoother Uplink Power Control Algorithm Based on Distance Ratios for Wireless Data Transfer Systems
}

\author{
Vadim ROMANUKE \\ Polish Naval Academy, Faculty of Navigation and Naval Weapons, \\ 69 Śmidowicza Street, Gdynia, 81-127, Poland \\ v.romanuke@amw.gdynia.pl
}

\begin{abstract}
A novel fast-and-smoother power control algorithm is suggested for wireless data transfer systems. It uses an adaptive multistep technique for equalizing the uplink quality of service. The technique is based on ratios of distances to the base station, and on the path loss exponent correctors if the farthest transmitters are occasionally assigned excessive powers. The constraint is a maximum-tolerated grand total of the powers transmitted in the uplink off all the transmitters. If the sum of the current uplink powers is not greater than that constraint, the powers are not corrected. Otherwise, they are corrected to fit in the active transmitter power interval by trying to equalize the powers received by the base station. If theoretic powers of the closest transmitters are too low, they are raised to the minimally possible transmitter power output. If theoretic powers of the farthest transmitters are too high, they are reduced to the maximally possible transmitter power output. The represented algorithm routine is fast enough to keep the UMTS update frequency $(1500 \mathrm{~Hz})$.
\end{abstract}

Keywords: Wireless data transfer, Power control, Quality of service, Interference, Uplink, UMTS.

\section{Introduction}

The modern world cannot exist without wireless data transfer (WDT). Systems providing WDT must comply with requirements which restrict the transmitter power output. The restriction is fundamentally needed because the overall spectral density of radio signals has been irreversibly growing and it will continue growing due to the increasing number of radio transmitters. The growth influences negatively not only WDT (in particular, telecommunication) as interference amongst different users, but also health (Sinha, Ghosh \& Sinha, 2015).

WDT systems use definite parts of the radio spectrum, which itself is a part of the electromagnetic spectrum with a range of frequencies from $30 \mathrm{~Hz}$ to $300 \mathrm{GHz}$ (Miao et al., 2016; Stojmenović, 2002). Since wireless phones and Wi-Fi networks have become widespread, the power of the generated and transmitted electromagnetic waves, called radio waves, has been intensively increasing in those definite parts (mainly, from the ultra high frequency band to the extremely high frequency band, that is from $300 \mathrm{MHz}$ up to $300 \mathrm{GHz}$ ) of this frequency range. To prevent interference between different users, the generation and transmission of radio waves is strictly regulated by national laws, coordinated by an international body, the International Telecommunication Union (ITU) (see, e. g., Stojmenović, 2002).

The ITU coordinates spectrum policy internationally. ITU is divided into three sectors determining the technical characteristics and operational procedures for wireless services, and playing a vital role in the spectrum management. ITU also manages the procedures of standardization and development. Spectrum management is a process of regulating the use of radio frequencies to promote efficient use and gain a net social benefit (Hossain \& Bhargava, 2007; Dahlman, Parkvall \& Sköld, 2011; Dahlman, Parkvall \& Sköld, 2018). The spectrum management considers also the increasing demand for WDT services. For instance, demand for wireless broadband has soared due to rapid expansion of $3 \mathrm{G} / 4 \mathrm{G}$ mobile services and the wireless internet services (Chuah \& Zhang, 2006; Dahlman, Parkvall \& Sköld, 2011; Kennington, Olinick \& Rajan, 2011).

Whereas the spectrum management dispatches radio frequencies, the power control is a process of optimizing amplitudes of those frequencies. Broadly speaking, this is an intelligent selection of the transmitter power output in a communication system to achieve good performance within the system (Hossain \& Bhargava, 2007; Dahlman, Parkvall \& Sköld, 2011; Díaz-Ibarra et al., 2019). Good performance for WDT services (which include both a system providing WDT and its users) mainly implies functioning without unwanted interference. This is the primary goal of the power control in communication networks (Hossain \& Bhargava, 2007; Lasaulce \& Tembine, 2011; Yao, 2018). Maintaining an admissible network capacity, geographic coverage and range, 
data transfer rate are secondary goals of the power control in WDT. These goals are closely tied to the primary goal of the (transmit) power control (Dahlman, Parkvall \& Sköld, 2018; Hossain \& Bhargava, 2007).

Addressing this background, the paper is organized as follows. The motivation for the research is stated in detail in Section 2. The paper's goal and tasks for its achievement are given in Section 3. Section 4 then describes principal parameters of the power control algorithm, which itself is stated in Section 5. Results of simulating the power control by the suggested algorithm are given in Section 6. The paper's findings, impact, and limitations are discussed in Section 7. Conclusions on the research are stated in Section 8.

\section{Motivation}

Basically, a substantial role in designing power control algorithms is played by either the base station or the wireless router, each of which is a transceiver connecting a number of devices to one another and/or to a wider area. The base station is the core of a WDT system. Thus, on the one side, the power control can be centralized, where the base station itself assigns transmitter power levels to users/mobiles considering their link qualities. On the other side, mobiles can be allowed to update their powers autonomously, considering their quality of service (QoS) perceived (Manvi, 2010). Apparently, in this way, the users become independent of the base station. Therefore, the mobiles can be considered as selfish agents (players) who try to maximize their utilities. Eventually, the non-cooperative game theory has been persistently used to model such multi-agent maximization processes (Hossain \& Bhargava, 2007; Lasaulce \& Tembine, 2011).

The latter directly refers to implementing the cognitive radio, which is dynamically programmed and configured in order to use the best wireless channels in its vicinity to avoid interference and congestion. Theoretically, along with trying to maximize the throughput and connectivity of the terminal/node the cognitive radio functions for, this system automatically detects available channels in the wireless spectrum. The cognitive radio then tries to adjust its transmission/reception parameters to allow more concurrent wireless communications in a given spectrum band at one location. Such a process is a form of dynamic spectrum management (Hossain \& Bhargava, 2007; Chuah \& Zhang, 2006).

At the same time, non-cooperative game theory models of wireless network power control claim that the selfish nodes try to maximize their own utility (i. e., throughput and connectivity) only as opposed to maximizing the overall performance of the WDT system they operate in. Namely, the potential interactions among those selfish nodes are modeled by the finite non-cooperative game (Hossain \& Bhargava, 2007; Lasaulce \& Tembine, 2011). Then, with a utility function assigned for each node, researchers in this field strive to determine the most stable and advantageous situation in the game.

Utility functions are chosen to achieve the required QoS. One of the most prominent examples is a utility function in the uplink power control problem in a single-cell CDMA system with $N$ nodes (active user transmitters) (see, e. g., Hossain $\&$ Bhargava, 2007). The utility of the $k$-th node transmitting a power $p_{k}$ (in watts) was defined as a function of $N$ powers $\left\{p_{k}\right\}_{k=1}^{N}$ transmitted:

$u_{k}\left(\left\{p_{j}\right\}_{j=1}^{N}\right)=\frac{L R}{M p_{k}} f\left(\gamma_{k}\right)$

by a signal-to-interference ratio

$\gamma_{k}=W R^{-1} h_{k} p_{k}\left(\sigma^{2}+\sum_{\forall j \in\{\overline{1, N}\} \backslash k\}\}} h_{j} p_{j}\right)^{-1}$

for the $k$-th node, where each node transmits $L$ information bits in frames (or packets) of $M$ bits at a rate $R$ (bits per second), $M>L, W$ is an available spread-spectrum bandwidth (in $\mathrm{Hz}$ ), $\sigma^{2}$ is the additive white Gaussian noise power at the receiver (in watts), $\left\{h_{k}\right\}_{k=1}^{N}$ is a set of path gains from the node to the base station, and

$f\left(\gamma_{k}\right)=\left(1-e^{-\gamma_{k} / 2}\right)^{M}$

is an efficiency function. The path gain for the $k$-th node is calculated based on the simple path loss model (Hossain \& Bhargava, 2007):

$h_{k}=0.097 d_{k}^{-4}, k=\overline{1, N}$,

where $\left\{d_{k}\right\}_{k=1}^{N}$ are distances in meters, at which the nodes are located from the base station. It is easy to see that the values of function (1) are measured in bits per Joule. 
Despite that the model with utility function (1) takes into consideration signal-to-interference ratio (2) by (3) and (4), involving all the nodes, this approach has a quite obvious series of problems and inconsistencies:

1. Rate $R$ is assumed to be equal for all the nodes.

2. The same packet capacity.

3. The transmission of the same number of information bits.

4. The distances between the nodes are not considered.

Even if the first three assumptions may still be accepted for some reasons, the latter impacts badly on estimating the interference. This is so because, in simpler words, the signal-tointerference ratio is indeed intended to measure the influence of the other $N-1$ nodes. At that, the neighboring nodes influence each other in a more negative manner. Obviously, the signal-tointerference ratio estimated by (2) makes sense only for two trivial cases:

1. $N=2$.

2. $N=3$ : the nodes are located equidistantly from each other, i. e. they are at the vertices of the equilateral triangle.

In all other cases, when $N>3$, the nodes physically cannot be equidistant from each other. Therefore, formula (2) can hardly estimate the signal-to-interference ratio, unless there are just two transmitters working with the same base station (a very rare occurrence).

There is another problem with that model. If $N=2$ then function (1) can be plotted for each of the two transmitters. Let

$$
\begin{aligned}
& M=80, L=64, W=10^{6}, \\
& R=10^{4}, \sigma^{2}=5 \cdot 10^{-15}, \\
& d_{1}=1000, d_{2}=500,
\end{aligned}
$$

and the maximal power transmitted is 2 watts. Figure 1 shows the utility of the farthest transmitter. As it can be seen, its topmost utility is achieved at very small powers $p_{1}$. Figure 2 shows the utility of the closest transmitter. Here a pretty weird thing is noticed: the topmost utility of the transmitter located twice closer to the base station actually does not depend on the power of the farthest transmitter. Moreover, the topmost utility of the closest transmitter is achieved at the smallest possible power $p_{2}$. For parameters (5) and distances (6), whichever the minimal value of $p_{2}$ is, that topmost utility is achieved at that value. Finally, the sum of those two utilities seems ever weirder (Figure 3): its maximal value is achieved at powers close to zero. Besides, if the farthest transmitter increases its power, its utility significantly decreases. The weirdest thing is that the decrement is hardly dependent on the farthest transmitter's power (the utility will be almost just the same whether it is on the maximal power or on the ten times reduced power!).

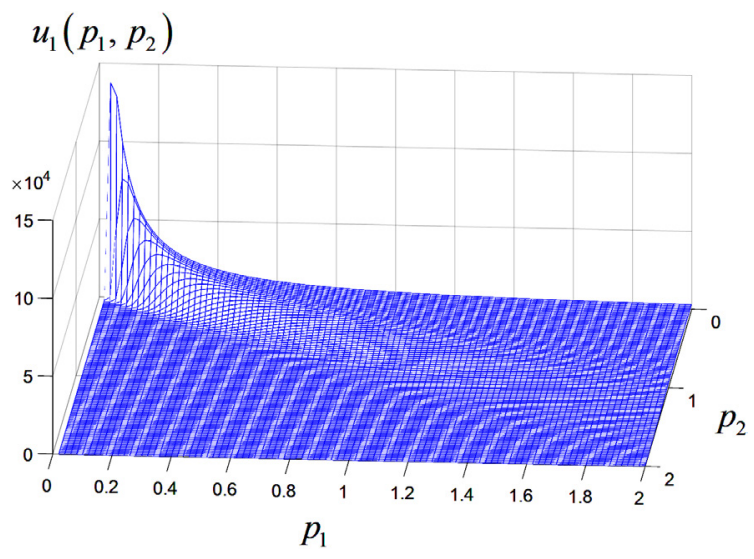

Figure 1. Utility $u_{1}\left(p_{1}, p_{2}\right)$ by model (1) - (4) for parameters (5) and distances (6)

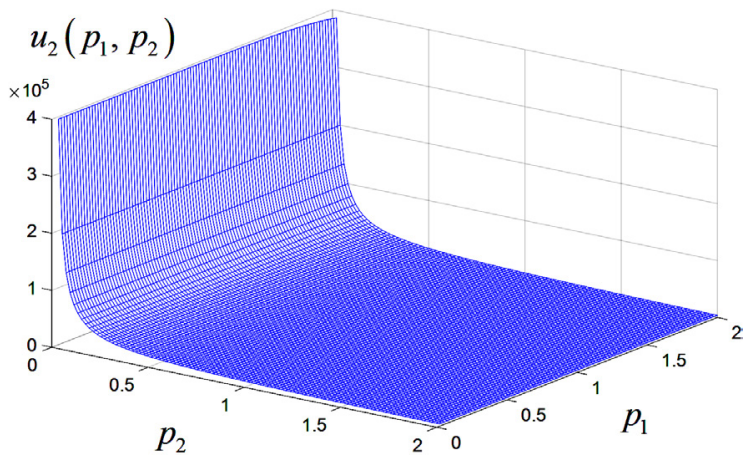

Figure 2. Utility $u_{2}\left(p_{1}, p_{2}\right)$ by model (1) - (4) for parameters (5) and distances (6)

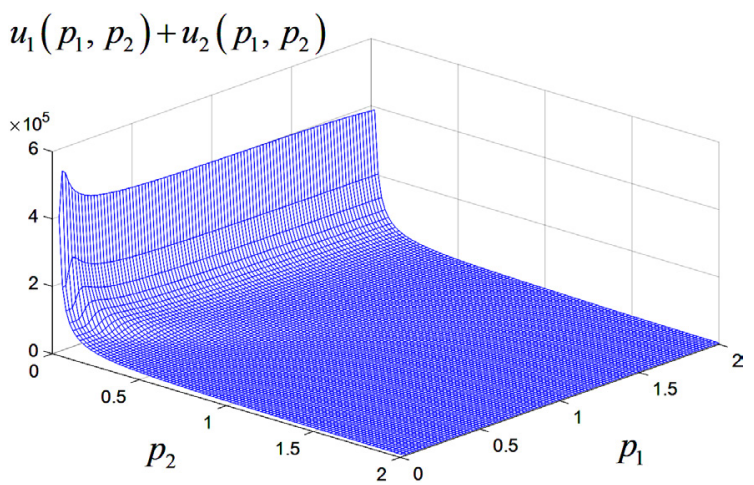

Figure 3. The sum of the utilities by model (1) - (4) for parameters (5) and distances (6)

Such inconsistent model cases occur for other ratios of distances $d_{1}, d_{2}$. But even if no attention 
is paid to their visualizations, the most obvious inconsistency is in the following: for any locations of the two transmitters, model (1) - (4) gives the same results by fixed distances $d_{1}$ and $d_{2}$ (Figure 4). So, implying the subsequent substantiation of the most favorable situation (either a Nash equilibrium or a Pareto point) for the WDT service, why should the model be so complicated?

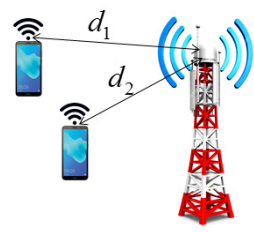

Figure 4. By the fixed distances from two transmitters to the base station, model (1) - (4) gives anyway the same signal-to-interference ratio for each transmitter, and the utilities are constant

The counterexample presented above is an absolute evidence of the fact that a technical implementation of cognitive radios requires distances between the nodes along with distances $\left\{d_{k}\right\}_{k=1}^{N}$. Meanwhile, the power control itself is a process of frequent corrections of powers transmitted for the base station. For instance, power control is executed 1500 times per second in the UMTS, which uses WCDMA technology to offer greater spectral efficiency and bandwidth to mobile network operators (Chuah \& Zhang, 2006; Kennington, Olinick \& Rajan, 2011; Sinha, Ghosh \& Sinha, 2015). Surely, it cannot be implemented using too complicated models, wherein re-calculation of utilities like (1) with its "inner" parts (2) - (4) and subsequent determination of the most favorable situation takes a way longer period than it would take for the centralized power control.

A far less sophisticated approach is used for radio resource management problems in 802.11 WLAN networks. Technically, the idea of the present approach is to automatically reduce the transmitted output power when other networks are within range. The levels of the power reduction are divided roughly (e. g., $1 \mathrm{~dB}, 2 \mathrm{~dB}, 3 \mathrm{~dB}$, as in the UMTS power control based on transmit power control commands received in the downlink). This is a primitive but nonetheless effective version of the cognitive radio (Chuah \& Zhang, 2006).

The motivation is a poor implementability of game-theory-based algorithms and rough (fast, though) centralized power control. The gametheory-based algorithms can fit WDT systems with slow power control (as in GSM cellular systems with their update frequency of $2 \mathrm{~Hz}$ ). The scale of the power reduction used in the UMTS is too rough, so its algorithm is not optimal. The question consists in the capability of a power control system to maintain the same power control frequency $(1500 \mathrm{~Hz})$ by using a smoother scale, which is believed to be derived from the estimated distances from the user transmitters to the base station.

\section{Goal and Tasks}

Due to the poor implementability and rough centralized power control, the goal is to equalize QoS by non-decreasing powers when moving away from the base station. For achieving this goal, the following tasks are to be accomplished:

1. Explain how and which powers and distances are estimated.

2. Argue for adjustable parameters.

3. State principal relationships of power correction for ensuring QoS.

4. Design an algorithm and its routine for power control based on those relationships.

5. Conduct simulation experiments and check whether power update frequency can be compared to that in the UMTS.

\section{Principal Parameters}

\subsection{Estimations of Powers and Distances}

Powers $\left\{p_{k}\right\}_{k=1}^{N}$ transmitted in the uplink are measured by user transmitters themselves. They are then sent to the base station in the nearest uplink transmission. Distances $\left\{d_{k}\right\}_{k=1}^{N}$ to the base station are estimated by a GPS navigation technique. They are sent to the base station along with the powers. Thus, each transmitter sends a pair of its current power and distance.

The distances are sorted in descending order by the base station:

$d_{i} \geqslant d_{i+1} \forall i=\overline{1, N-1}$. 
The powers are then re-indexed according to the sorted distances.

\subsection{Ratios of Distances by the Path Loss}

It is known that if a transmitter is distanced by $d$ meters from a receiver, then the path loss is estimated in $\mathrm{dB}$ as

$H=10 \mu \lg d$,

where $\mu$ is the path loss exponent (Hossain \& Bhargava, 2007). Values of this exponent are normally in the range of 2 to $4: \mu=2$ is for propagation of radio waves in free space, $\mu=4$ is for relatively lossy environments. In some environments (buildings, covered stadiums, etc.), the path loss exponent can reach values in the range of 4 to 6 .

Denote a nominal power (which is actually transmitted) by $p_{\text {nom }}$, and the received power by $p_{\text {rec }}$. Then path loss (8) can be re-written as

$H=10 \mu \lg d=10 \lg \left(p_{\text {nom }} p_{\text {rec }}^{-1}\right)$,

whence

$\lg d^{\mu}=\lg \left(p_{\text {nom }} p_{\text {rec }}^{-1}\right)$

and

$d^{\mu}=p_{\text {nom }} p_{\text {rec }}^{-1}$

So, the received power (at the base station) is

$p_{\text {rec }}=p_{\text {nom }} d^{-\mu}$.

Statement (10) implies that to ensure an equal QoS for a pair of transmitters distanced to the base station differently, not the distance between the transmitters, but the distance raised to the power of $\mu$ should be taken into account. This is why ratios of distances

$r_{i, i+1}=d_{i}^{\mu} d_{i+1}^{-\mu} \quad \forall i=\overline{1, N-1}$

by the path loss model (8) will be used further. Ratios (11) are calculated by the base station, which is assumed to "know" the path loss exponent over the area where this station operates.

\subsection{Adjustable Parameters}

Apart from the values of the powers meant to be transmitted in the uplink and the distances, there are also parameters which are adjustable. One of those adjustable parameters is the above-mentioned path loss exponent, which can be increased if the area where a base station operates becomes overbuilt with various architectural constructions. The other three ones are (all in watts):

1. A maximum-tolerated grand total $p_{\Sigma}$ of the powers transmitted in the uplink off all the transmitters.

2. A maximally possible transmitter power output $p_{\max }$.

3. A minimally possible transmitter power output $p_{0}$.

Whereas values $p_{\max }$ and $p_{0}$ are not presumed to be varied widely (like $p_{\max }=2$ and $p_{0}=0.001$ for most of cellular networks, although they are lower for the UMTS), $p_{\Sigma}$ depends on the capacity of the cell. Occasionally, $p_{\Sigma}$ can be expressed as the maximal number of user transmitters which could effectively simultaneously work within a scope of the same base station.

\section{Algorithm of Power Control}

\subsection{Relationships of Ensuring QoS}

Once the information about powers $\left\{p_{k}\right\}_{k=1}^{N}$ is received by the base station, it checks whether they not exceed the maximum-tolerated grand total:

$\sum_{k=1}^{N} p_{k} \leqslant p_{\Sigma}$

by

$p_{i} \geqslant p_{i+1} \forall i=\overline{1, N-1}$.

If (12) holds then the powers are not corrected, i. e.

$p_{k}^{*}=p_{k} \quad \forall k=\overline{1, N}$.

Otherwise, they are corrected to respective powers $\left\{p_{k}^{*}\right\}_{k=1}^{N}$ so, that

$\sum_{k=1}^{N} p_{k}^{*}=p_{\Sigma}$ 
by

$$
p_{k}^{*} \leqslant p_{\max } \quad \forall k=\overline{1, N}
$$

and

$$
p_{0} \leqslant p_{k}^{*} \forall k=\overline{1, N} \text {. }
$$

\subsection{Corrections of Powers}

As the primary goal is to ensure an equal QoS for all the users, the received power (10) should be the same. This means that, for the neighboring transmitters,

$p_{\text {rec }}=p_{i+1}^{*} d_{i+1}^{-\mu}=p_{i}^{*} d_{i}^{-\mu} \quad \forall i=\overline{1, N-1}$.

It follows from (18) that

$p_{i}^{*}=p_{i+1}^{*} d_{i}^{\mu} d_{i+1}^{-\mu}=r_{i, i+1} p_{i+1}^{*} \quad \forall i=\overline{1, N-1}$.

Let $\lambda$ be a correcting coefficient such that

$p_{N}^{*}=\lambda p_{N}$.

Next, using (19), every farther transmitter can be expressed by the closest one:

$p_{N-1}^{*}=r_{N-1, N} p_{N}^{*}=r_{N-1, N} \lambda p_{N}$,

$p_{N-2}^{*}=r_{N-2, N-1} p_{N-1}^{*}=r_{N-2, N-1} r_{N-1, N} \lambda p_{N}$,

and so on, including the power for the farthest transmitter

$p_{1}^{*}=r_{12} p_{2}^{*}=r_{12} r_{23} p_{3}^{*}=\ldots=$

$=r_{12} r_{23} r_{34} \ldots r_{N-2, N-1} p_{N-1}^{*}=$

$=r_{12} r_{23} r_{34} \ldots r_{N-2, N-1} r_{N-1, N} \lambda p_{N}$.

Then, using (15) and (20) - (23), the grand total is

$\sum_{k=1}^{N} p_{k}^{*}=\lambda p_{N}+r_{N-1, N} \lambda p_{N}+r_{N-2, N-1} r_{N-1, N} \lambda p_{N}+\ldots+$

$+r_{12} r_{23} r_{34} \ldots r_{N-2, N-1} r_{N-1, N} \lambda p_{N}=$

$=\lambda p_{N}\left(1+\sum_{i=1}^{N-1} \prod_{s=1}^{i} r_{N-s, N-s+1}\right)=p_{\Sigma}$,

where

$p_{N}^{*}=\lambda p_{N}=p_{\Sigma}\left(1+\sum_{i=1}^{N-1} \prod_{s=1}^{i} r_{N-s, N-s+1}\right)^{-1}$

$p_{j}^{*}=\left(p_{\Sigma} \prod_{s=1}^{N-j} r_{N-s, N-s+1}\right)\left(1+\sum_{i=1}^{N-1} \prod_{s=1}^{i} r_{N-s, N-s+1}\right)^{-1}$

$\forall j=\overline{1, N-1}$.

\subsection{Processing Exceptions and Special Cases}

As an exception, if

$N p_{0}>p_{\Sigma}$

holds, then the farthest-from-the-base-station

$N_{\text {off }}=N-\xi\left(p_{\Sigma} / p_{0}\right)$

transmitters whose distances are $\left\{d_{w}\right\}_{w=1}^{N_{\text {off }}}$, where function $\xi(x)$ returns the integer part of $x$, will be turned off:

$p_{w}^{*}=0 \quad \forall w=\overline{1, N_{\text {off }}}$ by $N_{\text {off }}<N$.

Then, using (25) and (26), only the powers of those transmitters which will not be turned off are corrected. Thus,

$N^{(\mathrm{obs})}=N$ and $N=\xi\left(p_{\Sigma} / p_{0}\right)$,

and powers $\left\{p_{l}\right\}_{l=N_{\text {off }}+1}^{N_{\text {(obs })}}$ are internally accepted for this exception as $\left\{p_{k}\right\}_{k=1}^{N}$; distances $\left\{d_{l}\right\}_{l=N_{\text {off }}+1}^{N^{(\mathrm{obs})}}$ are internally accepted for this exception as $\left\{d_{k}\right\}_{k=1}^{N}$.

The first special case is when (16) is violated. Then the $N_{\text {over }}$ farthest-from-the-base-station transmitters are obtained, for which

$p_{v}^{*}>p_{\max } \forall v=\overline{1, N_{\text {over }}}$ by $N_{\text {over }}<N$.

Their powers are set back at the maximum:

$p_{v}^{*}=p_{\max } \forall v=\overline{1, N_{\text {over }}}$ by $N_{\text {over }}<N$.

Subsequently, the rest of the powers, which are $\left\{p_{k}^{*}\right\}_{k=N_{\text {over }}+1}^{N}$ with their respective distances $\left\{d_{k}\right\}_{k=N_{\text {over }}+1}^{N}$, are corrected once again by

$p_{\Sigma}^{\text {(obs) }}=p_{\Sigma}, p_{\Sigma}=p_{\Sigma}^{\text {(obs) }}-p_{\text {max }} N_{\text {over }}$.

The second special case is when (17) is violated.

Then the $N_{\text {under }}$ closest-to-the-base-station transmitters are obtained, for which

$p_{u}^{*}<p_{0} \quad \forall u=\overline{N-N_{\text {under }}+1, N}$

by $N_{\text {under }}<N$. Their powers are set back at the minimum:

$p_{u}^{*}=p_{0} \quad \forall u=\overline{N-N_{\text {under }}+1, N}$.

Subsequently, the rest of the powers, which are $\left\{p_{k}^{*}\right\}_{k=1}^{N-N_{\text {under }}}$ with their respective 
distances $\left\{d_{k}\right\}_{k=1}^{N-N_{\text {under }}}$, are corrected once again by $p_{\Sigma}^{\text {(obs) }}=p_{\Sigma}, \quad p_{\Sigma}=p_{\Sigma}^{\text {(obs) }}-p_{0} N_{\text {under }}$.

\subsection{Path Loss Exponent Decrement}

If (16) is still violated after having processed the second special case, the path loss exponent is forced to be decreased. A local task here is to find a close-to-optimal $\mu^{*}$, a maximally possible value, at which (16) and (17) hold both. Such a task is going to be solved by means of a gradual decrement using an approach of dichotomization. So, let

$\mu_{\text {upper }}=\mu$ and $\mu_{\text {lower }}=\mu$.

Then $\mu_{\text {lower }}$ is decreased by some $\Delta \mu_{\text {dec }}$ until (16) and (17) hold both. This is a primary rough stage of determining the interval $\left(\mu_{\text {lower }} ; \mu_{\text {upper }}\right)$. The unknown $\mu^{*}$ lies between $\mu_{\text {lower }}$ and $\mu_{\text {upper }}$. Then the second stage goes. Firstly,

$\Delta \mu=\left(\mu_{\text {upper }}-\mu_{\text {lower }}\right) / 2$

and a new path loss exponent is

$\mu=\mu_{\text {lower }}+\Delta \mu$

The powers are recorrected again and

$\Delta \mu^{(\mathrm{obs})}=\Delta \mu, \Delta \mu=\Delta \mu^{(\mathrm{obs})} / 2$.

If (16) is still violated then

$\mu^{\text {(obs) }}=\mu$ and $\mu=\mu^{(\mathrm{obs})}-\Delta \mu$,

otherwise

$\mu^{\text {(obs) }}=\mu$ and $\mu=\mu^{(\mathrm{obs})}+\Delta \mu$.

\subsection{Main Routine}

The main routine operates on the known $p_{\Sigma}$, $p_{\max }, p_{0}, \mu$, and distances and powers from the current uplink, along with an accuracy $\Delta \mu_{0}$ :

${ }^{\mathrm{M}} 1$. Do subroutine \#A.

M2. If (16) is violated then do subroutine \#B; otherwise, return the corrected powers.

M3. Make definitions by (38) and (39).

M4. Re-correct powers by subroutine \#A using the path loss exponent by (39).
${ }^{\mathrm{M}} 5$. While $\Delta \mu>2^{-16}$, do subroutine \#C.

${ }^{\mathrm{M}} 6$. Return the finally corrected powers.

Subroutine \#A:

${ }^{\mathrm{A}} 1$. If (12) holds, then return the powers by (14).

A2. If (27) holds, then do subroutine \#1A.

A3. Correct powers by subroutine \#2A.

A4. Among the corrected powers, find such a set $I_{\text {over }}$ of their indices, for which (16) is violated.

A5. While set $I_{\text {over }}$ is nonempty, do subroutine \#3A.

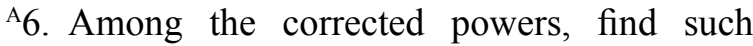
a set $I_{\text {under }}$ of their indices, for which (17) is violated.

A7. While set $I_{\text {under }}$ is nonempty, do subroutine \#4A.

${ }^{\mathrm{A}} 8$. Return the corrected powers.

Subroutine \#B:

${ }^{\text {B}} 1$. Make definitions by (37).

B2. While (16) is violated, decrease $\mu_{\text {lower }}$ by $\Delta \mu_{\text {dec }}$ and do subroutine \#A by setting $\mu=\mu_{\text {lower }}$.

Subroutine \#C:

${ }^{\mathrm{C}} 1$. Make the definition by (40).

c2. If (16) is violated, make the definition by (41); otherwise, if $\Delta \mu<\Delta \mu_{0}$ then break the subroutine, else make the definition by (42).

C3. Do subroutine \#A.

Subroutine \#1A:

${ }^{1 \mathrm{~A}} 1$. Find (28) and drop powers to (29).

${ }^{1 \mathrm{~A}} 2$. Make definitions by (30). 
1A3. Accept powers $\left\{p_{l}\right\}_{l=N_{\text {off }}+1}^{N_{\text {(obs })}}$ as $\left\{p_{k}\right\}_{k=1}^{N}$.

1A4. Accept $\left\{d_{l}\right\}_{l=N_{\text {off }}+1}^{N^{(\mathrm{obs})}}$ as $\left\{d_{k}\right\}_{k=1}^{N}$.

Subroutine \#2A:

${ }^{2 \mathrm{~A}} 1$. Calculate ratios (11).

${ }^{2 \mathrm{~A}} 2$. Calculate powers (25) and (26).

Subroutine \#3A:

${ }^{3 \mathrm{~A}} 1$. Drop powers to (32).

${ }^{3 A} 2$. Re-correct the rest (non-dropped) powers $\left\{p_{k}^{*}\right\}_{k=N_{\text {over }}+1}^{N}$ with their respective distances $\left\{d_{k}\right\}_{k=N_{\text {over }}+1}^{N}$ for (33) by subroutine \#2A.

Subroutine \#4A:

${ }^{4 \mathrm{~A}} 1$. Raise powers to (35).

${ }^{4 \mathrm{~A}} 2$. Re-correct the rest (non-raised) powers $\left\{p_{k}^{*}\right\}_{k=1}^{N-N_{\text {under }}}$ with their respective distances $\left\{d_{k}\right\}_{k=1}^{N-N_{\text {under }}}$ for (36) by subroutine \#2A.

\section{Simulation Experiments}

For simulation experiments, an example of a single-carrier UMTS cell by $p_{\max }=2$ watts, $\mu=4, \Delta \mu_{0}=2^{-10}$ is taken. Simulation power control results are shown in Figures $5-8$, wherein all powers are given in watts. Only Figure 5 presents an example of a single step algorithm performance by no dropped or raised powers (the execution time is $0.49 \mathrm{~ms}$ ). On the contrary, Figure 6 contains an example where the powers are raised to 0.05 watts (the execution time is $0.51 \mathrm{~ms}$ ). Figure 7 presents an example in which the powers are dropped to 2 watts (the execution time is $0.56 \mathrm{~ms}$ ). An example of an "overcrowded" cell is shown in Figure 8, wherein a majority of powers are raised to 0.05 watts, and 123 transmitters are turned off (the execution time

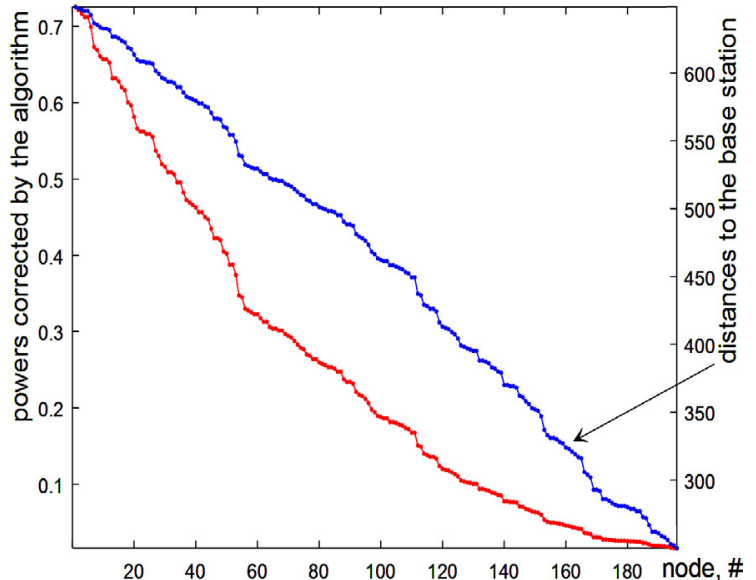

Figure 5. A simulation power control by 196 nodes, performed in a single step for $p_{\Sigma}=49, p_{0}=0.01$

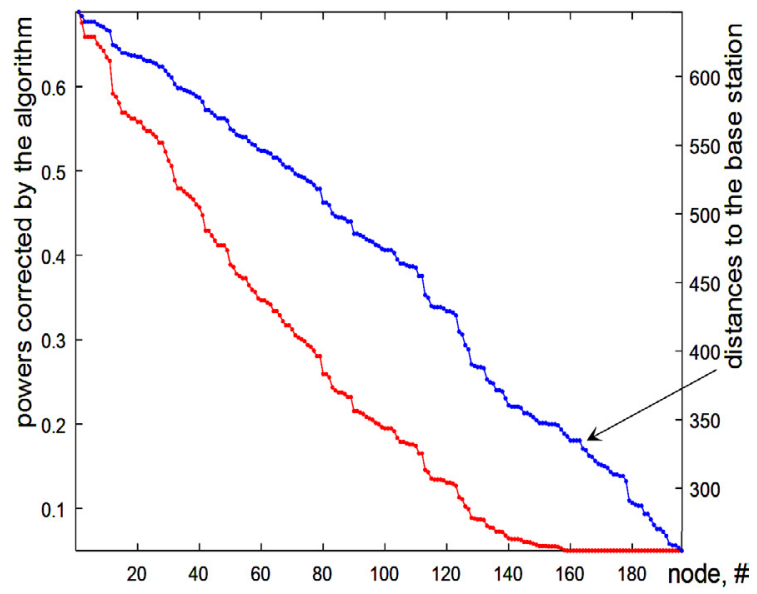

Figure 6. A simulation power control by 196 nodes, performed in 3 steps for $p_{\Sigma}=49, p_{0}=0.05$

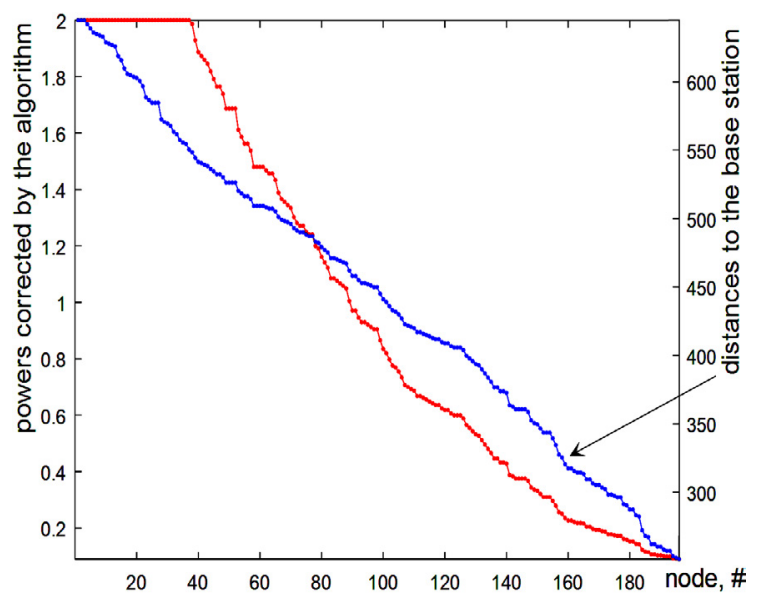

Figure 7. A simulation power control by 196 nodes, performed in 4 steps for $p_{\Sigma}=196, p_{0}=0.05$ is $0.62 \mathrm{~ms}$ ). 


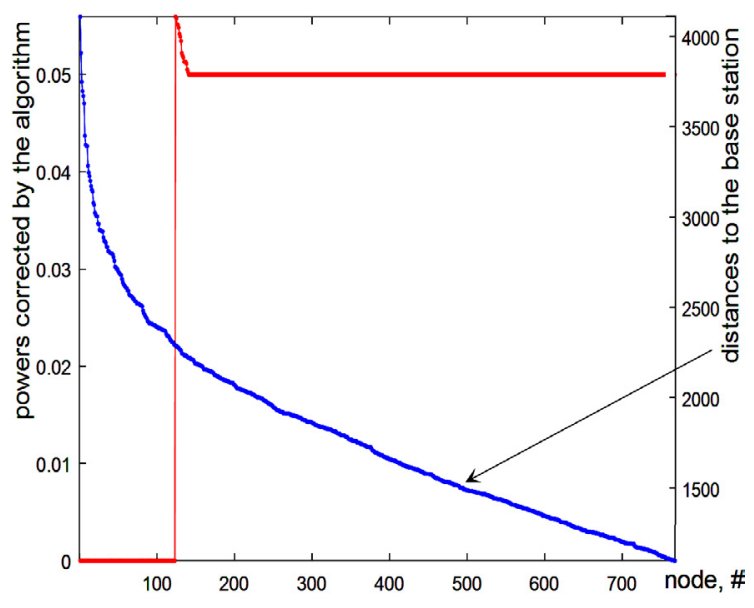

Figure 8. A simulation power control by 768 nodes, performed in 8 steps for $p_{\Sigma}=32.3, p_{0}=0.05$

\section{Discussion}

The QoS equalization is as important as preventing interference amongst users of the WDT service. In fact, they influence each other. Having radially measured distances to the base station, the uplink powers are controlled and corrected in order to receive as many equal powers as possible. It is a conception of ensuring equitable access for users to WDT services.

Whereas non-cooperative game theory models of power control are too "slow and heavy", the suggested approach is based on distance ratios (11), and on the path loss exponent correctors if the farthest transmitters are occasionally assigned excessive powers. This approach is in total compliance with the existing inner loop power control, wherein the base station sends commands received by the user's node in the downlink to adjust its power at the next uplink signal. The represented routine is fast enough to keep the UMTS update frequency $(1500 \mathrm{~Hz})$.

\section{REFERENCES}

1. Chuah, M. C. \& Zhang, Q. (2006). Design and Performance of $3 G$ Wireless Networks and Wireless LANs. Springer.

2. Dahlman, E., Parkvall, S. \& Sköld, J. (2011). Power Control, Scheduling, and Interference Handling. $4 G$ LTE/LTEAdvanced for Mobile Broadband, 265-299. Academic Press.

3. Dahlman, E., Parkvall, S. \& Sköld, J. (2018). Uplink Power and Timing Control, 5 G NR: the
Distances between transmitters are ignored. It seems to be a fair lack as thus interference is poorly (implicitly) estimated. Introducing such "interdistances" into the signal-to-interference ratio would improve much credibility of (18) - (26). However, accurate measurement of these "interdistances" is hardly realizable due to the finite accuracy of GPS. Besides, it will take a way longer periods to update the powers within a WDT system with a few hundred users.

\section{Conclusion}

This paper suggests a novel power control algorithm with using an adaptive multistep technique for equalizing the uplink QoS. The algorithm performs a fast-and-smoother uplink power distribution that can be implemented into WDT systems with frequent power updates as in the UMTS. Once a table of power levels is defined, where rounding to a specific number of digits in decimal fractions is maintained, the base station controls the power of the transmitter by sending a power level tag/number. The transmitter then adjusts its uplink power accordingly.

The suggested algorithm is a smooth centralized power control meant for improving QoS when moving away from the base station. It admits a case of turning down transmitters "overcrowding" the WDT system. The turn-down is short-term owing to a high update frequency $(1500 \mathrm{~Hz})$.

\section{Acknowledgements}

The research for this paper was financially supported by the Institute of Naval Weapons and Informatics of the Faculty of Navigation and Naval Weapons at the Polish Naval Academy, Gdynia, Poland.

Next Generation Wireless Access Technology, 301-310. Academic Press.

4. Díaz-Ibarra, M. A., Campos-Delgado, D. U., Gutiérrez, C. A. \& Luna-Rivera, J. M. (2019). Distributed power control in mobile wireless sensor networks, $A d$ Hoc Networks, $85,110-119$.

5. Hossain, E. \& Bhargava, V. K. (2007). Cognitive Wireless Communication Networks. Springer. 
6. Kennington, J., Olinick, E. \& Rajan, D. (2011). Wireless Network Design. Optimization Models and Solution Procedures. Springer.

7. Lasaulce, S. \& Tembine, H. (2011). EnergyEfficient Power Control Games, Game Theory and Learning for Wireless Networks, 183-209. Academic Press.

8. Manvi, S. S. (2010). Wireless and Mobile Networks: Concepts and Protocols. Wiley.

9. Miao, G., Zander, J., Sung, K. W. \& Slimane, B. (2016). Fundamentals of Mobile Data Networks. Cambridge University Press.
10. Sinha, K., Ghosh, S. C. \& Sinha, B. P. (2015). Wireless Networks and Mobile Computing. CRC Press.

11. Stojmenović, I. (2002). Handbook of Wireless Networks and Mobile Computing. Wiley.

12. Yao, J., Lou, W., Yang, C. \& Wu, K. (2018). Efficient interference-aware power control for wireless networks, Computer Networks, $136,68-79$. 